\title{
Microglia lacking a peroxisomal $\beta$-oxidation enzyme chronically alter their inflammatory profile without evoking neuronal and behavioral deficits
}

Lien Beckers ${ }^{1,6}$, Ivana Geric ${ }^{1}$, Stijn Stroobants², Sander Bee ${ }^{3,4}$, Philip Van Damme ${ }^{3,4,5}$, Rudi D'Hooge ${ }^{2}$ and Myriam Baes ${ }^{1 *}$ (D)

\begin{abstract}
Background: Microglia play a central role in most neurological disorders, but the impact of microgliosis on brain environment and clinical functions is not fully understood. Mice lacking multifunctional protein-2 (MFP2), a pivotal enzyme in peroxisomal $\beta$-oxidation, develop a fatal disorder characterized by motor problems similar to the milder form of MFP2 deficiency in humans. The hallmark of disease in mice is the chronic proliferation of microglia in the brain, but molecular pathomechanisms that drive rapid clinical deterioration in human and mice remain unknown. In the present study, we identified the effects of specific deletion of MFP2 from microglia in the brain on immune responses, neuronal functioning, and behavior.
\end{abstract}

Methods: We created a novel CX3cr1-Mfp2 ${ }^{-/-}$mouse model and studied the impact of MFP2 deficiency on microglial behavior at different ages using immunohistochemistry and real-time PCR. Pro- and anti-inflammatory responses of $\mathrm{Mfp}^{-/-}$microglia were assessed in vitro and in vivo after stimulation with IL-1 $\beta /$ INFY and IL-4 (in vitro) and LPS and IL-4 (in vivo). Facial nerve axotomy was unilaterally performed in $\mathrm{C} \times 3 \mathrm{Cr} 1-\mathrm{Mfp}^{-/-}$and control mice, and microglial functioning in response to neuronal injury was subsequently analyzed by histology and real-time PCR. Finally, neuronal function, motor function, behavior, and cognition were assessed using brainstem auditory evoked potentials, grip strength and inverted grid test, open field exploration, and passive avoidance learning, respectively.

Results: We found that $\mathrm{Mfp}^{-2^{--}}$microglia in a genetically intact brain environment adopt an inflammatory activated and proliferative state. In addition, we found that acute inflammatory and neuronal injury provoked normal responses

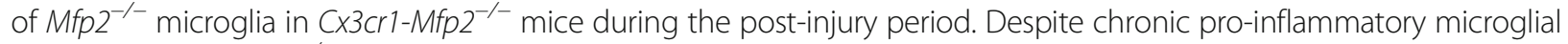
reactivity, $\mathrm{C} \times 3 \mathrm{cr} 1-\mathrm{Mfp}^{-/-}$mice exhibited normal neuronal transmission, clinical performance, and cognition.

Conclusion: Our data demonstrate that MFP2 deficiency in microglia causes intrinsic dysregulation of their inflammatory profile, which is not harmful to neuronal function, motor function, and cognition in mice during their first year of life.

Keywords: Microglia, Peroxisomes, Facial nerve axotomy, Conditional mouse model, $\beta$-Oxidation, Behavior, Immune response

\footnotetext{
* Correspondence: Myriam.Baes@kuleuven.be

${ }^{1}$ Department of Pharmaceutical and Pharmacological Sciences, Laboratory

for Cell Metabolism, KU Leuven - University of Leuven, Campus Gasthuisberg

O/N2, Herestraat 49, B-3000 Leuven, Belgium

Full list of author information is available at the end of the article
}

(c) The Author(s). 2019 Open Access This article is distributed under the terms of the Creative Commons Attribution 4.0 International License (http://creativecommons.org/licenses/by/4.0/), which permits unrestricted use, distribution, and reproduction in any medium, provided you give appropriate credit to the original author(s) and the source, provide a link to the Creative Commons license, and indicate if changes were made. The Creative Commons Public Domain Dedication waiver (http://creativecommons.org/publicdomain/zero/1.0/) applies to the data made available in this article, unless otherwise stated. 


\section{Background}

Inactivation of peroxisomal $\beta$-oxidation by the loss of multifunctional protein-2 (MFP2) in human and mice causes a fatal neuropathological phenotype [1-4]. MFP2, encoded by the $H s d 17 b 4$ gene, is the key enzyme in peroxisomal $\beta$-oxidation, a pathway responsible for chain shortening of carboxylates including very long chain fatty acids and formation of polyunsaturated fatty acids [5]. Dependent on the type of mutation, patients with MFP2 (also called D-bifunctional protein) deficiency display a severe neurodevelopmental disorder leading to death within the first year of life or a milder phenotype with prolonged survival into adolescence or adulthood [3, 6, 7]. Prominent clinical presentations of the milder phenotype are sensorineural hearing loss, leukodystrophy, intellectual decline, ataxia, and sensorimotor neuropathy $[3,8,9]$. Most symptoms are mimicked by the constitutive $M f p 2^{-/}$mouse model which develops a progressive fatal phenotype characterized by motor problems, ataxia, weight loss, and lethargy $[1,2]$. The pathomechanisms of disease and role of MFP2 in the brain remain however elusive in human and mice.

The most prominent hallmark of $M f p 2^{-/-}$mice is a strong neuroinflammatory response consisting of proliferating resident microglia in the absence of neuronal loss $[2$, 10, 11]. Characterization of this excessive microgliosis in the brain of $M f p 2^{-/-}$mice revealed that resident microglia proliferate, adopt a permanently activated non-phagocytic state, and lose their homeostatic signature $[10,11]$. Specific suppression of microgliosis in $M f p 2^{-/-}$mice by treatment with PLX5622, a selective colony-stimulating factor 1 receptor (CSF1R) inhibitor, failed to prevent neuronal dysfunction and clinical deterioration of $M f p 2^{-/-}$mice as inflammatory responses and residual reactive microglia remained after treatment [12]. The importance of peroxisomal $\beta$-oxidation in innate immune cells is poorly understood, but $M f p 2^{-/-}$mice do not show systemic inflammation, and there is no infiltration of peripheral immune cells in the brain [10].

Microglia, the primary immune effector cells in the brain, can rapidly respond to disturbances of central nervous system (CNS) homeostasis by adopting an inflammatory activation state which consists of morphological alterations, proliferation, upregulation of cell surface markers, and increased expression of inflammatory molecules [13-15]. The so-called guardians of the brain adopt resting and activated states depending on the brain environment or the insult. Chronic activation of microglia was assumed to be detrimental to proper CNS functioning, but microglial activation is in fact a delicately balanced process that constitutes both harmful and protective effects [16-18].

This early-onset aberrant phenotype of $M f p 2^{-/-}$microglia gains even more interest as we previously defined that microglia in Nestin-Mfp $2^{-/-}$mice develop a late-onset and mild inflammatory state [11]. The neural-specific Nestin-
$M f p 2^{-/-}$mouse model lacks MFP2 in neurons, astrocytes, and oligodendrocytes but not in microglia [2]. The chronic and strongly activated microglial phenotype in constitutive $M f p 2^{-/}$mice was associated with early-onset deficits in neuronal transmission, explorative behavior, and cognition. In contrast, attenuated microgliosis in Nestin-Mfp $2^{-/-}$mice was associated with late-onset and minor abnormalities in neuronal function and behavior compared to $M f p 2^{-/-}$mice. Whereas constitutive $M f p 2^{-/-}$mice die within 4-6 months, Nestin-Mfp $2^{-/-}$mice survive up to 8-12 months [2, 19]. Although the progression of microgliosis parallels clinical deterioration, it remains unknown whether the dysregulated microglial phenotype and the behavioral abnormalities are caused by cell-autonomous MFP2 dysfunction in microglia.

Therefore, to investigate the importance of MFP2 function within microglia, we generated a novel mouse model that lacks MFP2 specifically in myeloid cells by $C \times 3 c r 1$-driven recombination of the $H s d 17 b 4$ gene [20]. In the brain parenchyma, the chemokine receptor CX3CR1 is exclusively expressed by microglia [21, 22]. We characterized the $C \times 3 c r 1-M f p 2^{-/}$mice with regard to the morphological and immunological properties of microglia and examined responses of $M f p 2^{-/-}$microglia in vitro and in vivo to immunological challenges and neuronal injury by facial nerve axotomy. In addition, the impact of microglia-specific deletion of MFP2 on neuronal functioning and murine behavior and cognition was assessed. Our study demonstrated that microglia-specific deletion of MFP2 from the Cx3cr1-Mfp2 $2^{-/}$brain leads to intrinsic alterations of microglia that develop a pro-inflammatory and proliferative phenotype but retain proper responses to inflammatory stimuli. This chronic adaptation of $M f p 2^{-/-}$microglia in a genetically intact brain did however not affect neuronal transmission or murine motor function, cognition, and explorative behavior within the time frame wherein all $M f p 2^{-/-}$mice have died from the disease [19].

\section{Methods}

Mouse breeding

$M f p 2^{\text {loxP/loxP }}$ mice in which exon 8 of the $H s d 17 b 4$ gene is flanked by LoxP sequences [2] were bred with Cx3cr1-Cre mice, which cause recombination in brain microglia, monocytes, subsets of natural killer, and dendritic cells [20], on a C57Bl6 background. $M f p 2^{-/-}$mice were obtained by breeding heterozygous mice as described [2]. Genotyping was performed on ear punches. All mice were bred in the animal housing facility of the KU Leuven, had ad libitum access to water and standard rodent food, and were kept on a 12-h light and dark cycle.

\section{Murine behavioral studies}

The auditory brainstem response test (BAEP), the open field (OF) exploration, and the grip strength measurement 
were conducted as previously described [11]. Passive avoidance (PA) learning was examined in a cage consisting of a light and a dark compartment containing a grid floor [23]. After a 30-min adaptation to the dark, the mouse was placed in the light compartment for a training trial. After $5 \mathrm{~s}$, the dark compartment was opened and step-through latency was manually recorded. When all paws were placed on the grid floor, a mild electric footshock $(0.2 \mathrm{~mA}, 2 \mathrm{~s})$ was applied. Retention was tested $24 \mathrm{~h}$ later in the dark-adapted mouse, and latency to enter the dark compartment was measured up to a 300-s cutoff value. The inverted grid test or four-limb hang test is a test of combined forepaw and hind paw strength and coordination. Mice are placed on a wired grid which is subsequently inverted. The latency to fall is recorded with a time limit of $300 \mathrm{~s}$. In general, normal mice are able to remain on the inverted grid for at least $300 \mathrm{~s}$. Mice that fall off the grid before the time limit of $300 \mathrm{~s}$ were directly given another try, and the best time was recorded. Mice that hang for the 300-s limit were placed back into the cage.

\section{Administration of lipopolysaccharide}

Mice aged 5 months and 8 months received an intraperitoneal (i.p.) injection of lipopolysaccharide (LPS) at a dose of $1 \mathrm{mg} / \mathrm{kg}$ (Sigma, L4391) or sterile saline vehicle in a total volume of $100 \mu \mathrm{l}$. Four hours later, mice were sacrificed, and brainstem was collected and flash frozen in liquid nitrogen for subsequent RNA analysis.

\section{Intracerebroventricular injection of IL-4}

The IL-4 injections were performed on 5-month-old mice as described previously [24]. Briefly, mice were anesthetized with ketamine and xylazine (100 and 10 $\mathrm{mg} / \mathrm{kg}$, respectively) and injected with vehicle $(0.9 \%$ $\mathrm{NaCl})$ or $200 \mathrm{ng}$ IL-4 (R\&D) in a total volume of $2.5 \mu \mathrm{l}$ in the third cerebral ventricle using the following stereotaxic coordinates: bregma, $-0.25 \mathrm{~mm}$; lateral, $1 \mathrm{~mm}$; and depth, $2.25 \mathrm{~mm}$. The animals were allowed to recover for $20 \mathrm{~h}$, after which they were sacrificed with an overdose of Domitor and Nimatek $(1 \mathrm{mg}$ and $75 \mathrm{mg} / \mathrm{kg}$, respectively). The frontal cortex contralateral to the injection site was collected and snap frozen in liquid nitrogen.

\section{Facial nerve axotomy}

The facial nerve injury experiment was performed as described [25]. Mice were 3-month-old at the time of the surgical procedure. In brief, mice were anesthetized with $3 \%$ isoflurane and placed on a $37^{\circ} \mathrm{C}$ hot plate during the surgical procedure. Unilateral facial nerve transection at the stylomastoid foramen, posterior from the retroauricular branch point, was performed in Cx3cr1-Mfp $2^{-/-}$ and control mice. The successful outcome of the procedure was verified by ipsilateral whisker paresis immediately upon recovery from mild anesthesia. The facial nerve motor nucleus at the ipsi- and contralateral side of the injury was collected from frozen sections as described [25]. For RNA extraction, the PicoPure RNA isolation kit (Thermo Fisher Scientific) was used.

\section{Immunohistochemical staining and quantification}

Mice were anesthetized with a mix of Domitor $(1 \mathrm{mg} /$ $\mathrm{kg}$ ) and Nimatek $(75 \mathrm{mg} / \mathrm{kg})$. Tissue processing and IHC staining were performed as described [1, 26, 27]. Briefly, mice were perfused transcardially with PBS ( $\mathrm{pH} 7.4$ ) followed by $4 \%$ paraformaldehyde (PFA). The brains were isolated, post-fixed with $4 \%$ PFA overnight, and kept in $70 \%$ ethanol prior to paraffin embedding. Paraffin sections $(7 \mu \mathrm{m})$ were used for immunofluorescent stainings with polyclonal rabbit anti-Iba1 (1:500; Wako D19-19741) or rat anti-F4/80 (1:500; Serotec, Oxford, UK). For detection, HRP-labeled secondary antibodies (1:200) and fluorescent labeling with a cyanine 2 (FITC) TSA kit (Perkin Elmer Life Sciences, Boston, USA) were used. Cell nuclei were labeled with Vectashield Antifade Mounting Medium with DAPI (Vector Laboratories, United Kingdom). Images were acquired with a motorized inverted IX-81 microscope connected to a CCD-FV2T digital camera (Olympus, Aartselaar, Belgium) and processed with LSM Image Browser software (Zeiss, Germany).

Microglial cell numbers were counted around the sagittal midline and coronal plane at the height of brainstem and visual cortex. Within one plane $(\times 20$ magnification), only Iba1-positive cells that (1) had fully co-localized with DAPI-positive nuclei, (2) had a clear cell soma, and (3) had at least two clear protrusions were counted in different regions of the brain. Microglial number per frame was corrected for surface area. Three to five different pictures per brain region per mouse were taken. The number of microglia was counted, and the average of all pictures per brain region was used to quantify the number of microglial cells per brain region ( $n=4-6$ /group).

For the facial nerve axotomy model, brains were, after transcardial perfusion (described above), incubated in $30 \%$ sucrose in phosphate-buffered saline (PBS) at $4{ }^{\circ} \mathrm{C}$ until fully submerged. All tissues were protected from light. Samples were embedded in OCT (Tissue-Tek) for frozen sectioning on a cryostat (Leica). Cryosections from brains with unilateral lesions were prepared on slides and kept at $-20^{\circ} \mathrm{C}$ until use. Tissues were rehydrated or permeabilized in blocking solution $(0.1 \%$ Triton-X 100, 5\% bovine albumin, normal goat serum, and PBS) for $1 \mathrm{~h}$ at room temperature and then incubated overnight at $4{ }^{\circ} \mathrm{C}$ with primary antibody diluted in $5 \%$ serum and PBS. The following primary antibodies were used: polyclonal rabbit anti-Iba1 (1:500; Wako 019-19,741) and rat anti-F4/80 (1:500; Serotec, Oxford, UK). Extensive wash steps were performed with PBS, and 
sections were incubated with goat anti-rabbit secondary antibody conjugated to Alexa Fluor 488 (1:1000; Life Technologies A32731) for $1 \mathrm{~h}$ at room temperature. Microglia responses were analyzed 1 day and 5 days post-axotomy. Iba $1^{+}$fluorescence intensity and numbers of microglia cells per area were measured in ipsi- and contralateral sides, and the ratio of ipsilateral side relative to their respective contralateral side is shown for both genotypes (Cx3cr1-Mfp $2^{-/-}$and control mice).

\section{Microglia isolation and cell culture}

Microglial cells were isolated from control and $M f p 2^{-/-}$ pups at postnatal day 8 (P8) using magnetic-activated cell sorting (MACS) according to the manufacturer's instructions (Miltenyi Biotec). Cells were plated in 12-well plates in a Macrophage Serum Free Medium (Thermo Fisher) and stimulated for $24 \mathrm{~h}$ with $50 \mathrm{ng} / \mathrm{ml} \mathrm{IL1} \beta$ and $20 \mathrm{ng} / \mathrm{ml} \mathrm{IFN} \gamma$ or $50 \mathrm{ng} / \mathrm{ml} \mathrm{IL-4}$ (all from R and D) to induce a pro- and anti-inflammatory phenotype, respectively.

For confirming recombination of the $M f p 2$ gene, microglia were isolated from 11-month-old control and Cx3crl$M f p 2^{-/-}$mice. Mice were anesthetized with a mix of Domitor $(1 \mathrm{mg} / \mathrm{kg})$ and Nimatek $(75 \mathrm{mg} / \mathrm{kg})$ and perfused with approximately $20 \mathrm{ml}$ ice-cold HBSS (without calcium and magnesium). Subsequently, brains were removed and dissociated to a single cell suspension using the Neural Tissue Dissociation Kit (P) according to the manufacturer's instructions for the automated dissociation using the gentleMACS Dissociator (Miltenyi Biotec). Next, myelin was removed by $22 \%$ Percoll gradient and the cell suspension was further processed for microglia separation. Microglia (positive fraction) were separated from other brain cells (negative fraction) by MACS following the manufacturer's instructions using CD11b MicroBeads (Miltenyi Biotec). Both the positive and negative fractions were further processed for qRT-PCR.

\section{Real-time quantitative PCR}

Total RNA was isolated from snap-frozen brain tissue using Trizol reagent (Invitrogen, California, USA) or from isolated or cultured microglia by using the PureLink RNA Mini Kit, both according to the manufacturer's protocol. Subsequently, cDNA was generated from a 1- $\mu$ g RNA using the QuantiTect Reverse Transcription Kit (QIAGEN, Venlo, The Netherlands). For real-time PCR, an ABI PRISM 7500 Real-Time PCR instrument (Applied Biosystems, Lennik, Belgium) was used. Primers and probes were ordered from Applied Biosystems as premade Taqman Gene Expression Assays (Il1b, Mm011336189_m1; Cx3cr1, Mm0262011_s1; Tgfbr1, Mm00436964_m1; arginase 1, Mm00475991_m1; Mrc1, Mm00485148_m1) and used as previously described [27]. Alternatively, the following genes were tested in triplicate using the PowerUp SYBR
Green Master Mix (Thermo Fisher) with primers ordered from Integrated DNA Technologies (Leuven, Belgium): cd200, Mm.PT.58.33215550; Cx3cl1, Mm.PT.58.8767901; Csf1r, Mm.PT.58.12811749; Csf1, Mm.PT.58.11661276; Cxcl1, Mm.PT.58.8767901; Il34, Mm.PT.58.32379406; Fizz-1, Mm.PT.58.43062398; Il4, Mm.PT.58.7882098; Ym-1, Mm.PT.58.33370435; Tlr2, Mm.PT.58.45820113; Tspo, Mm.PT.58.43313736; F4/80 or Emr1, Mm.PT.58 .11087779; Tmem119, Mm.PT.58.6766267; P2ry12, Mm. PT.58.43542033; and Mfp2, Mm.PT.58.16985875. The data were analyzed using the $2-\Delta \Delta C \mathrm{~T}$ method [28]. The relative expression levels of the target genes were calculated as a ratio to the housekeeping gene $\beta$-actin except for the facial nerve injury experiment for which adaptorrelated protein complex 3 , delta 1 subunit $(A p 3 d 1)$, F-box protein 38 (Fbxo38), and MON2 homolog (Mon2) were used that remain unaltered after neuronal injury [25]. The following primers were used: Ap3d1 (forward, 5'-CAAG GGCAGTATCGACCGC-3'; reverse, 5'-GATCTCGTC AATGCACTGGGA- 3'), Mon2 (forward, 5'-CTAC AGTCCGACAG GTCGTGA-3'; reverse, 5'-CGGCAC TGGAGGTTCTATATCTC-3'), and Fbxo38 (forward, 5'-ATGGGACCACGAAAG AAAAGTG-3'; reverse, 5'-T AGCTTCCGAGAGAGGCATTC-3').

\section{MFP2 activity measurements}

The forebrain of 11-month-old mice was homogenized in $5 \mathrm{mM}$ MOPS pH 7.2, $1 \mathrm{mM}$ EDTA, $250 \mathrm{mM}$ sucrose, and $0.1 \%(v / v)$ ethanol. After the appropriate dilution of the samples, the dehydratase activity of MFP2 was measured as previously described [29] with 3S-hydroxy-3-phenylproprionyl-CoA as a substrate, except that Thesit was increased to $0.05 \%(w / v)$.

\section{Statistical analysis}

All data except some behavioral tests (see below) were analyzed with GraphPad Prism software (version 5.0 and 6.0, San Diego, CA). Statistical analyses were carried out using unpaired and paired, two-sided Student's $t$ test, one-way ANOVA, two-way ANOVA, or two-way repeated measure (RM) ANOVA followed by the Bonferroni post hoc test. Data are shown as mean \pm standard error of the mean (SEM), and statistical significance was set at $p<0.05$. SPSS Statistics software was used for three-way ANOVA and three-way RM ANOVA.

\section{BAEP test}

Two-way ANOVA with genotype and (inter)peak as sources of variation was used to evaluate neuronal transmission in Cx3cr1-Mfp $2^{-/}$mice. The Holm-Sidak and Bonferroni methods were used for multiple comparisons. Unpaired $t$ test was used to evaluate the peak amplitudes. 


\section{OF and PA test}

Independent sample $t$ test and Mann-Whitney $U$ test were used to compare performance between different genotypes.

\section{Results}

Generation of microglia/monocyte-specific Cx3cr1-Mfp $2^{-/-}$ mice

In order to investigate whether inactivation of peroxisomal $\beta$-oxidation in microglia impacts on microglial behavior and may contribute to the neuropathology that we observed in $M f p 2^{-/-}$mice, we generated a microglia/ monocyte-specific knockout, by crossbreeding Cx3cr1Cre mice [20] with floxed $M f p 2$ mice [2]. First, we analyzed whether insertion of the Cre-recombinase gene in the genome and haploinsufficiency of CX3CR1 did not negatively influence microglial behavior in the brain. Microglia were investigated in the brain of both Cre-positive and Cre-negative control mice at 5, 8, and 12 months of age. No differences in $\mathrm{Iba}^{+}$cells regarding morphology and cell numbers were observed in the brain of Cre-positive (Cre Mfp $2^{\text {Wt/LoxP }}$ ) versus Cre-negative $\left(M f p 2^{W t / L o x P}\right)$ control mice (Additional file 1: Figure S1). There were no signs of microglial reactivity, and the F4/80 marker was absent in both Cre-positive and Cre-negative control mice at all ages (data not shown). In addition, no differences in GFAP expression were observed in mice of both genotypes indicating that insertion of Cre did not influence astroglia (data not shown). Therefore, we used both Cre-positive and Cre-negative mice as control animals. Because reliable antibodies for immunohistochemical detection of MFP2 are not available [30], we confirmed the recombination of $M f p 2$ in microglia of $C \times 3 c r 1-M f p 2^{-\prime}$ - mice using transcript analysis on MACS-isolated microglia. The selectivity of $M f p 2$ recombination was further confirmed by the normal expression of MFP2 in the non-microglia fraction and by the fact that the activity of MFP2 was not significantly reduced in whole brain homogenates (Additional file 2: Figure S2). Cx3cr1-Mfp $2^{-/-}$ mice were indistinguishable from their control littermates during the first year of life, were fertile and survived past the age of 14 months.

\section{Development of microglial proliferation and morphological transformation in the brain of $\mathrm{C} \times 3 \mathrm{cr} 1$ - $\mathrm{Mfp}^{2^{-/-}}$mice}

Constitutive $M f p 2^{-/-}$mice develop severe and extensive microgliosis from 6 weeks of age that progressively increases. In contrast, Nestin-Mfp $2^{-/-}$mice only develop microgliosis from 12 to 17 weeks of age, which is never as extensive as in constitutive $M f p 2^{-/-}$mice [11], suggesting that intrinsic loss of MFP2 from microglia might be involved in the development of microgliosis. In order to address this, we analyzed cell numbers and morphology of microglia in $C x 3 c r 1-M f p 2^{-/-}$mice at different ages. IHC analysis and quantification of the microglial marker Iba1 revealed that numbers of microglial cells progressively increased from the age of 3 months in all brain regions, both in gray and white matter, of $C \times 3 c r 1-M f p 2^{-/-}$mice compared to age-matched control mice (Fig. 1a-l). In the control mice, microglial cell numbers were similar across all ages that were investigated, so the data were combined (Fig. $1 \mathrm{k}, \mathrm{l}$ ). The proliferation of microglial cells was associated with progressive morphological transformation (Fig. $1 \mathrm{~m}-\mathrm{q}$ ). At 3 months of age, several microglia in the Cx3cr1-Mfp $2^{-/-}$ brain developed thicker and shorter protrusions and a mildly enlarged cell soma (Fig. 1n). These hypertrophic features became gradually more pronounced with age (Fig. 1o-q).

Microglial proliferation is typically induced by the cytokines colony-stimulating factor 1 (CSF1) or interleukin-34 (IL-34) that are ligands of the CSF1R. We analyzed whether the gene expression of these cytokines was changed in the brain of $C \times 3 c r 1-M f p 2^{-/-}$mice by qPCR analysis. Transcript levels of Csf1 were not altered (Fig. 1r), but transcript levels of Il34 and Csf1r were induced (Fig. 1s, t) in 8-month-old Cx3cr1-Mfp $2^{-/-}$mice in comparison to age-matched control mice.

In the healthy brain, neurons chronically restrain microglia in order to maintain their surveilling state and prevent microglial proliferation. To investigate whether the microglial proliferation was related to the loss of neuronal restraint signals, we analyzed pivotal markers in the brains of Cx3cr1-Mfp $2^{-/-}$mice. We found that transcript levels of Cx3cl (fractalkine) (Fig. 1u) and Cd200 (Fig. 1v) were equal to those in age-matched control brains. Taken together, the deletion of the peroxisomal $\beta$-oxidation enzyme MFP2 from microglia induces progressive morphological changes and IL-34-driven proliferation, typical features of microglial activation. In comparison to constitutive $M f p 2^{-/}$mice, the microgliosis is less pronounced and delayed in Cx3cr1-Mfp $2^{-/-}$brain $[10,11]$.

\section{Microglia in CX3cr1-Mfp2 ${ }^{-/}$mice are inflammatory activated and adopt a pro-inflammatory state at later stages}

The microglia/macrophage marker F4/80 becomes upregulated on the microglial membrane when microglia get activated in response to the disruption of CNS homeostasis, such as neuronal injury, aging, or infectious pathogens in the brain parenchyma. Numbers of activated $\mathrm{F} 4 / 80^{+}$ microglia increased with age in $C \times 3 c r 1-M f p 2^{-/-}$mice during the first year of life (Fig. 2a-f), whereas $\mathrm{F} 4 / 80^{+}$cells were absent in the control mice at all ages (Fig. 2a). Microglial activation is accompanied with the induction of inflammatory markers in pathological conditions. The transcript levels of the pro-inflammatory markers Tnfa, 

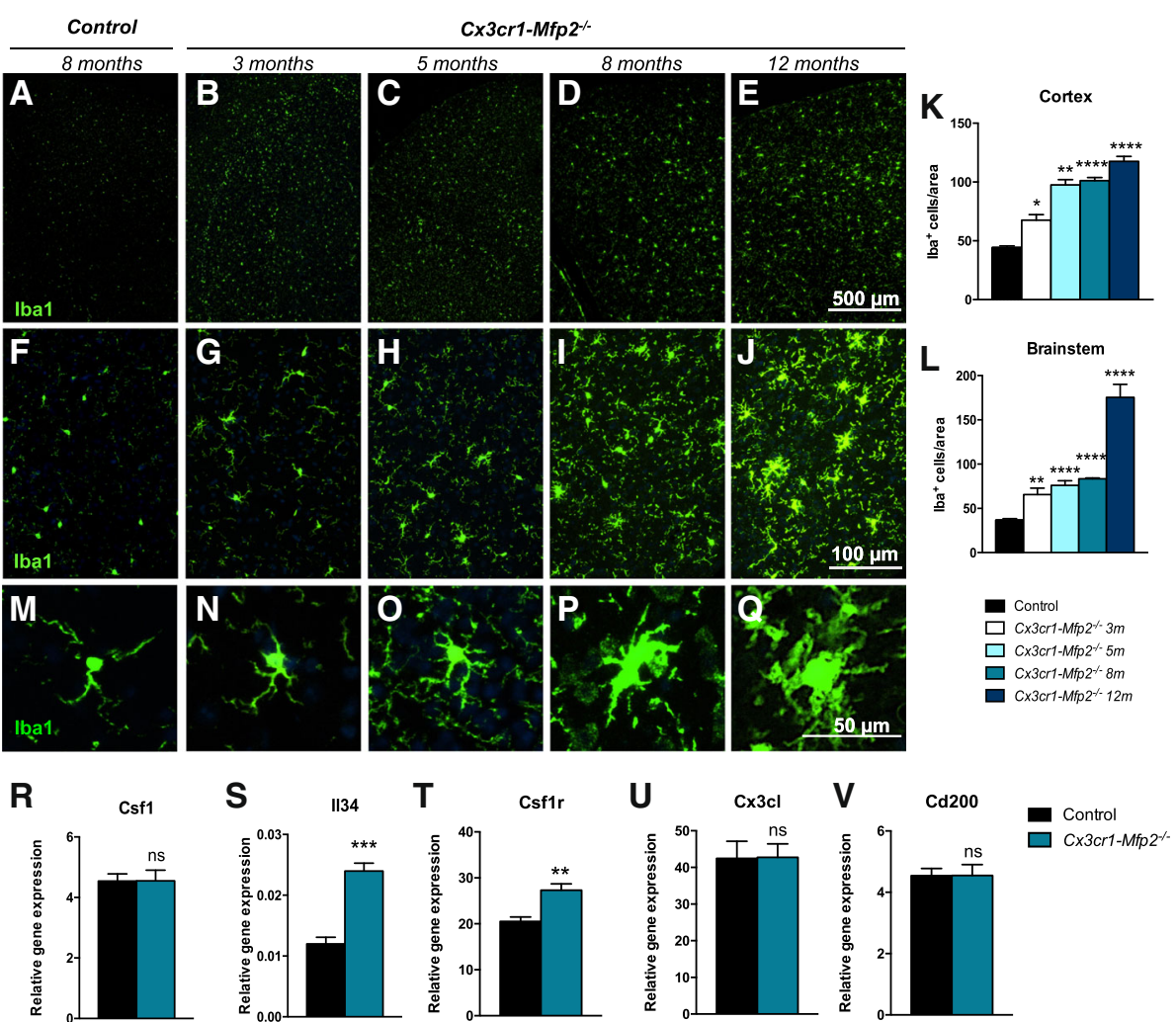

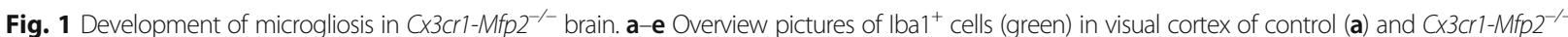
mice ( $\mathbf{b}-\mathbf{e})$ at the indicated ages and at higher magnification ( $\mathbf{f}-\mathbf{j}) \cdot \mathbf{k}, \mathbf{I}$ Quantification of microglial cells in the cortex (visual and motor cortex) and the brainstem of CX3cr1-Mfp2 ${ }^{-1-}$ mice ( $n=4$ mice/age) compared to control mice. Data of control mice across different ages $(3,5,8,12$ months) is combined ( $n=16$ mice). $\mathbf{m}-\mathbf{q}$ Gradual morphological transformation of microglia in $C \times 3 c r 1-M f p 2^{-1-}$ brain at the same ages. $\mathbf{r}-\mathbf{v}$ Gene expression analysis by qRT-PCR of markers related to the proliferation of microglia in 8-month-old mice. CX3cr1-Mfp2 ${ }^{-/-}$mice compared to control: ${ }^{* *} p<0.05,{ }^{* * *} p<0.001,{ }^{* * *} p<0.0001$. ns, not significant. Error bars indicate SEM. m, months. $n=4$ mice/group. Representative pictures are shown

$I l 1 b$, and $T l r 2$ were not significantly changed at 5 months of age but were induced at 8 months of age in $C \times 3 \mathrm{cr} 1$ $M f p 2^{-/-}$mice (Fig. $2 \mathrm{~g}-\mathrm{i}$ ). We assessed whether induction of pro-inflammatory markers was associated with the downregulation of anti-inflammatory markers. Transcripts of Il4 (Fig. 2j) and Fizz (Fig. 2k) were downregulated at 8 months, but not at 5 months of age. Transcript levels of Ym1 (Fig. 2l) and Arginase-1 (Arg1) (Fig. 2m) were not significantly decreased. Together, this indicates that $M f p 2^{-/-}$microglia in C $x 3 c r 1-M f p 2^{-/-}$mice adopt an activated pro-inflammatory phenotype.

Normal responses of $M f p 2^{-/-}$microglia to pro- and antiinflammatory challenges in vivo and in vitro

We subsequently assessed how microglia in Cx3cr1-Mfp $2^{-/-}$ mice respond to pro- and anti-inflammatory stimuli. LPS was systemically administered to 5-month-old (Fig. 3a, b) and 8-month-old (Fig. 3c, d) mice, and transcript levels of pro-inflammatory markers were monitored. Expression of Tnfa (Fig. 3a, c) and Illb (Fig. 3b, d) were similarly induced in the $C \times 3 c r 1-M f p 2^{-/-}$and control mice after LPS injection at both ages, indicating that microglia in Cx3cr1-Mfp2 $2^{-/-}$ mice are not primed. Subsequently, we investigated responses to an anti-inflammatory stimulus by performing i.c.v. injections of IL-4 in the Cx3cr1-Mfp $2^{-/-}$and control mice at 5 months of age. Both the $C \times 3 c r 1-M f p 2^{-/-}$and control mice exhibited an anti-inflammatory brain environment post-injection, evident by increased expression of antiinflammatory markers (Arg1 and Fizz1) (Fig. 3e, f) and unaltered expression of pro-inflammatory markers (Tnf, Illb, and $T l r 2$, data not shown). The data demonstrate normal responsiveness of $M f p 2^{-/-}$microglia to an anti-inflammatory challenge as anti-inflammatory markers in Cx3cr1-Mfp $2^{-/-}$ mice were elevated to the same extent as in control mice.

Finally, we tested whether cultured $M f p 2^{-/-}$microglia derived from P8 $M f p 2^{-/-}$mice react normally to cytokine exposure. Under basal conditions, no differences in expression levels of pro- or anti-inflammatory markers were observed (Additional file 3: Figure S3). Likewise, when challenged with a pro- or anti-inflammatory stimulus, $M f p 2^{-/-}$microglia responded similarly as compared to the control microglia (Additional file 3: Figure S3). Taken together, our results show that $M f p 2^{-/-}$microglia are not primed and respond normally to pro- and anti-inflammatory stimuli. 


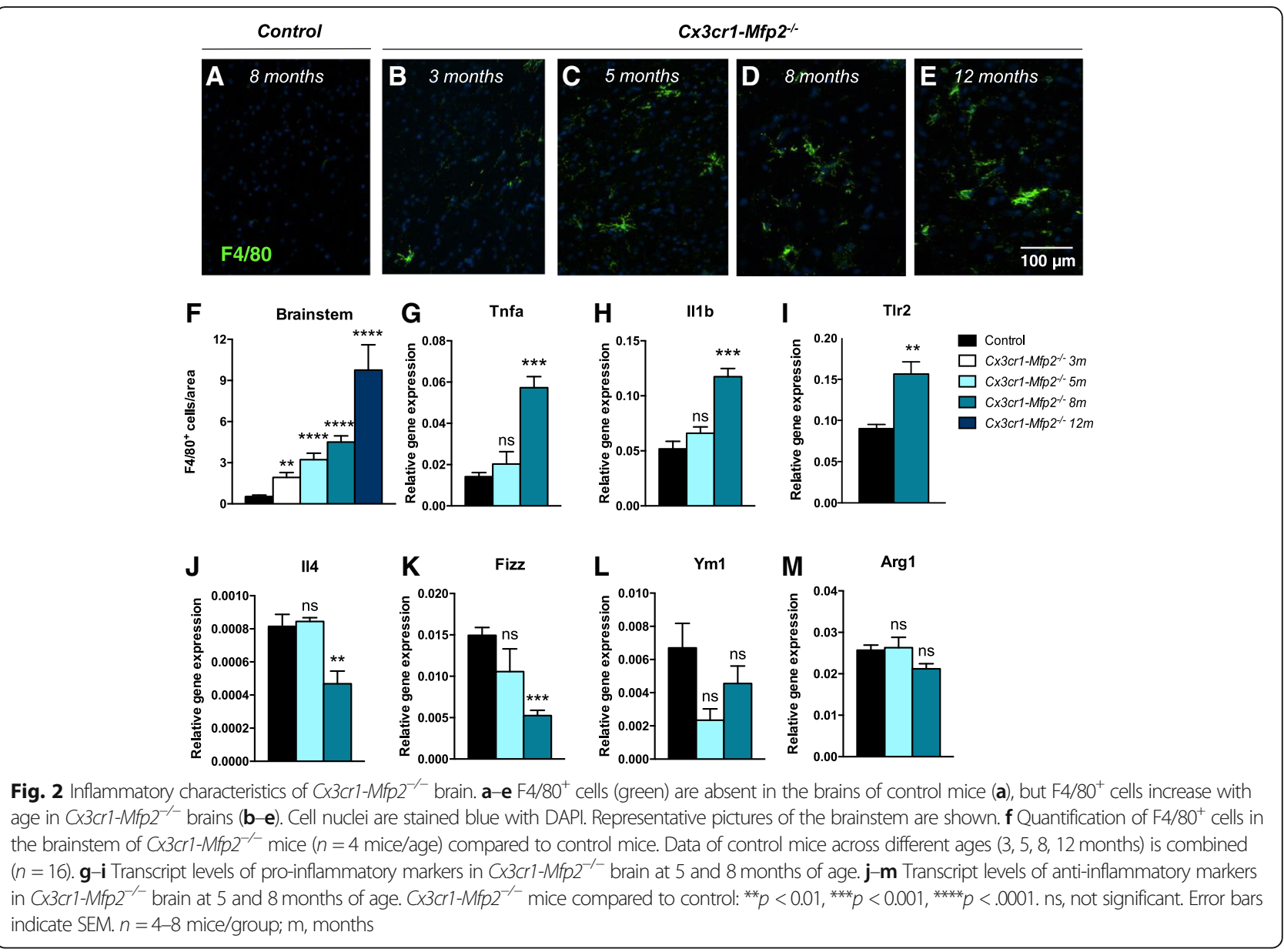

\section{$\mathrm{Mfp}^{-/-}$microglia in $\mathrm{C} \times 3 \mathrm{cr} 1-\mathrm{Mfp}^{-/-}$mice exhibit a normal response to neuronal injury}

In order to elucidate how $M f p 2^{-1-}$ microglia respond to neuronal injury, we induced unilateral transection of the facial nerve (FN) in 3-month-old C $\times 3 c r 1-M f p 2^{-/-}$and control mice. The contralateral side remained intact and was considered as the control side. Facial nerve axotomy provokes a local microglial response in the facial motor nucleus (FMN) in the brainstem [31-33] which reaches a maximum at 5 days post-injury [25]. The microglial response in FMN was analyzed at day 1 (Fig. $4 \mathrm{a}-\mathrm{j}$ ) and at day 5 post-axotomy (Fig. $4 \mathrm{k}-\mathrm{t}$ ). As microglia are activated and cell numbers increased in intact 3-month-old C $\times 3$ cr $1-M f p 2^{-/-}$relative to control brains (Fig. 1), Iba $1^{+}$ fluorescence intensity and cell numbers in ipsilateral FMNs were measured relative to their contralateral FMNs (ratio) in both genotypes. Iba1+ intensity and cell numbers were similarly increased in $C \times 3 c r 1-M f p 2^{-/-}$and control mice after facial nerve axotomy at day 1 (Fig. 4e, j) and at day 5 post-axotomy (Fig. 4o, t). At day 1 post-injury, fold change measurements of $\mathrm{Iba}^{+}$fluorescence in ipsilateral FMN relative to contralateral FMN indicated that microglial response to acute neurodegeneration is similar in
Cx3cr1-Mfp $2^{-/-}$(1.6-fold) and control (1.4-fold) mice, in parallel with similarly increased microglial numbers in ipsilateral FMN in control (1.5-fold) and Cx3cr1-Mfp $2^{-/-}$ (1.7-fold) mice. This indicates that $M f p 2^{-/-}$microglia respond normally to neuronal injury immediately after lesion was generated.

At 5 days post-axotomy, an extensive microglial response was generated in the ipsilateral FMN of both control (Fig. 4l, q) and Cx3cr1-Mfp $2^{-/-}$(Fig. 4n, s) mice. We found that there was no significant difference in the induction of microglial response in the ipsilateral FMN of control (16-fold) and Cx3cr1-Mfp $2^{-/-}$(13-fold) mice (Fig. 4o). Accordingly, microglial numbers are equally increased in the ipsilateral FMN of control (5.1-fold) and Cx3cr1-Mfp $2^{-/-}$(4.3-fold) mice (Fig. 4t). Taken together, the neuronal injury did not provoke an exaggerated or diminished inflammatory response in $M f p 2^{-/-}$microglia during the acute postinjury period. Accordingly, no differences were observed in F4/80 ${ }^{+}$microglial cells and $\mathrm{GFAP}^{+}$astroglial cells in the ipsilateral FMNs of $C x 3 c r 1-M f p 2^{-/-}$and control brain at day 1 and 5 post-axotomy (data not shown).

To assess the inflammatory profile of proliferated microglia, FMN at contra- and ipsilateral sides was 
A

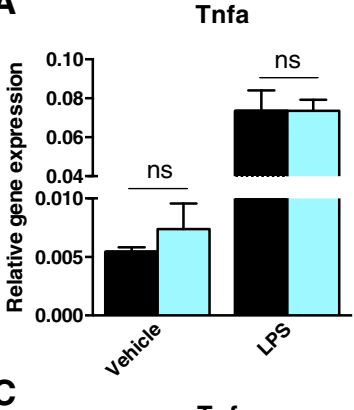

C

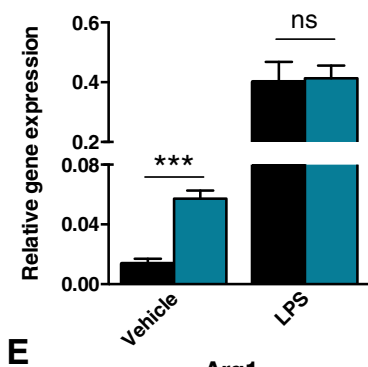

E

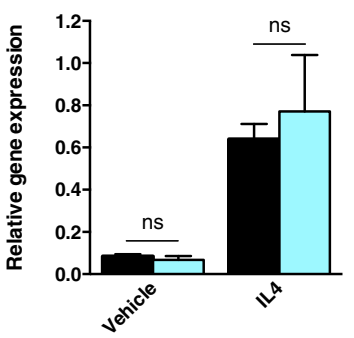

B

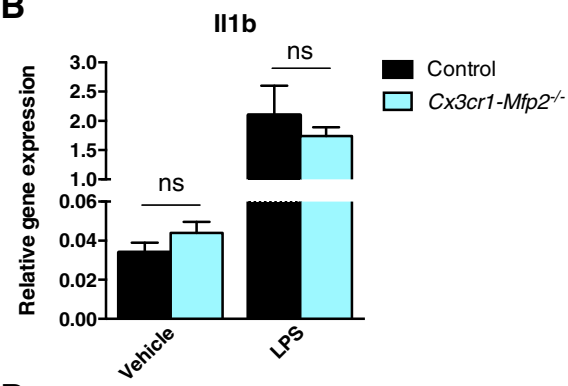

D

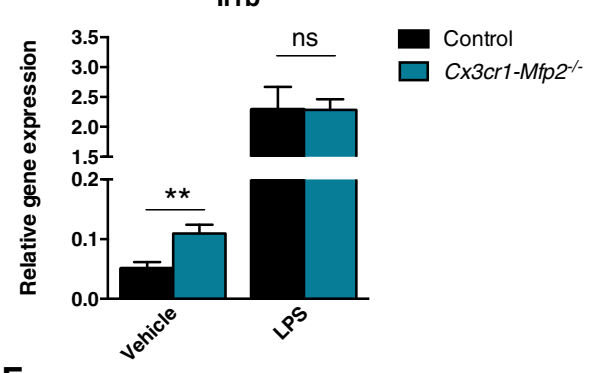

F

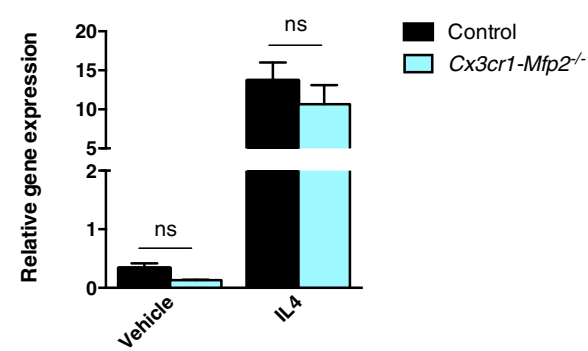

Fig. $3 \mathrm{C} \times 3 \mathrm{cr} 1-\mathrm{Mfp}^{-1-}$ mice respond adequately to pro- and anti-inflammatory challenges. a-d Five-month-old (a, b) and 8-month-old (c, d) $\mathrm{C} \times 3 \mathrm{cr} 1-\mathrm{Mfp} 2^{-/-}$ and control mice were challenged with i.p. LPS or vehicle, and the brainstem was analyzed after $6 \mathrm{~h}$ for transcript levels of pro-inflammatory markers. $n=4-6$ mice/group. e, $\mathbf{f}$ Five-month-old $\mathrm{Cx} 3 \mathrm{cr} 1-\mathrm{Mfp}^{-1-}$ and control mice were challenged with i.c.v. IL-4 or vehicle, and the frontal cortex contralateral to the injection site was analyzed after $20 \mathrm{~h}$ for transcript levels of anti-inflammatory markers. $n=3$ mice/group. Cx3cri-Mfp $2^{-1-}$ mice compared to control: ${ }^{* *} p<0.05$, ${ }^{* * *}$ $p<0.001$. ns, not significant. Error bars indicate SEM

isolated at 5 days post-axotomy from $C x 3 c r 1-M f p 2^{-/-}$ and control brain, and transcript levels of several inflammatory molecules were measured by qPCR analysis. In basal conditions, represented by the contralateral side, only the transcript levels of $I b a 1$ were significantly induced in Cx3crl-Mfp $2^{-/-}$compared to control mice. At the axotomized ipsilateral side, the expression of inflammatory markers being the microglial marker Iba1 (Fig. 5a); activation marker F4/80 (Fig. 5b); neuroinflammatory marker Tspo (Fig. 5c); pro-inflammatory markers Tlr2, Tnfa, and Il1b (Fig. 5d, e, f); proliferative microglial receptor Csf1r (Fig. 5g); and homeostatic microglial marker Tgfbr1 (Fig. 5h) were similarly induced versus the intact contralateral FMNs in both genotypes. These results show that $M f p 2^{-/-}$microglia in the Cx3cr1-Mfp $2^{-/-}$ brain exhibit a normal inflammatory reaction to acute neuronal injury.
Cx3cr1-Mfp2 $2^{-/-}$mice exhibit intact neuronal functioning and a normal clinical phenotype and cognition

We found previously that neuronal transmission of auditory signals in the brain was severely delayed and peak amplitudes were reduced in constitutive $M f p 2^{-/-}$mice after evoking auditory potentials in the brainstem (BAEPs). Decreased neuronal transmission progressed in parallel with aggravating neuroinflammation [11]. In contrast, there was only a minor delay in transmission and normal peak responses of evoked auditory signals in Nestin-Mfp $2^{-/}$ mice [11]. In order to reveal whether a pro-inflammatory state of microglia in C $x 3 \mathrm{cr} 1-\mathrm{Mfp} 2^{-/-}$mice affects auditory brainstem responses, BAEPs were analyzed in 8-month-old $C \times 3 c r 1-M f p 2^{-/-}$and control mice. Peak latencies show the time when the auditory signal evokes a response in a specific auditory nucleus in the brain [34]. Mean peak and interpeak latencies were similar in $C x 3 c r 1-M f p 2^{-/-}$and control mice (Fig. 6a,b), and peak amplitudes are normal 


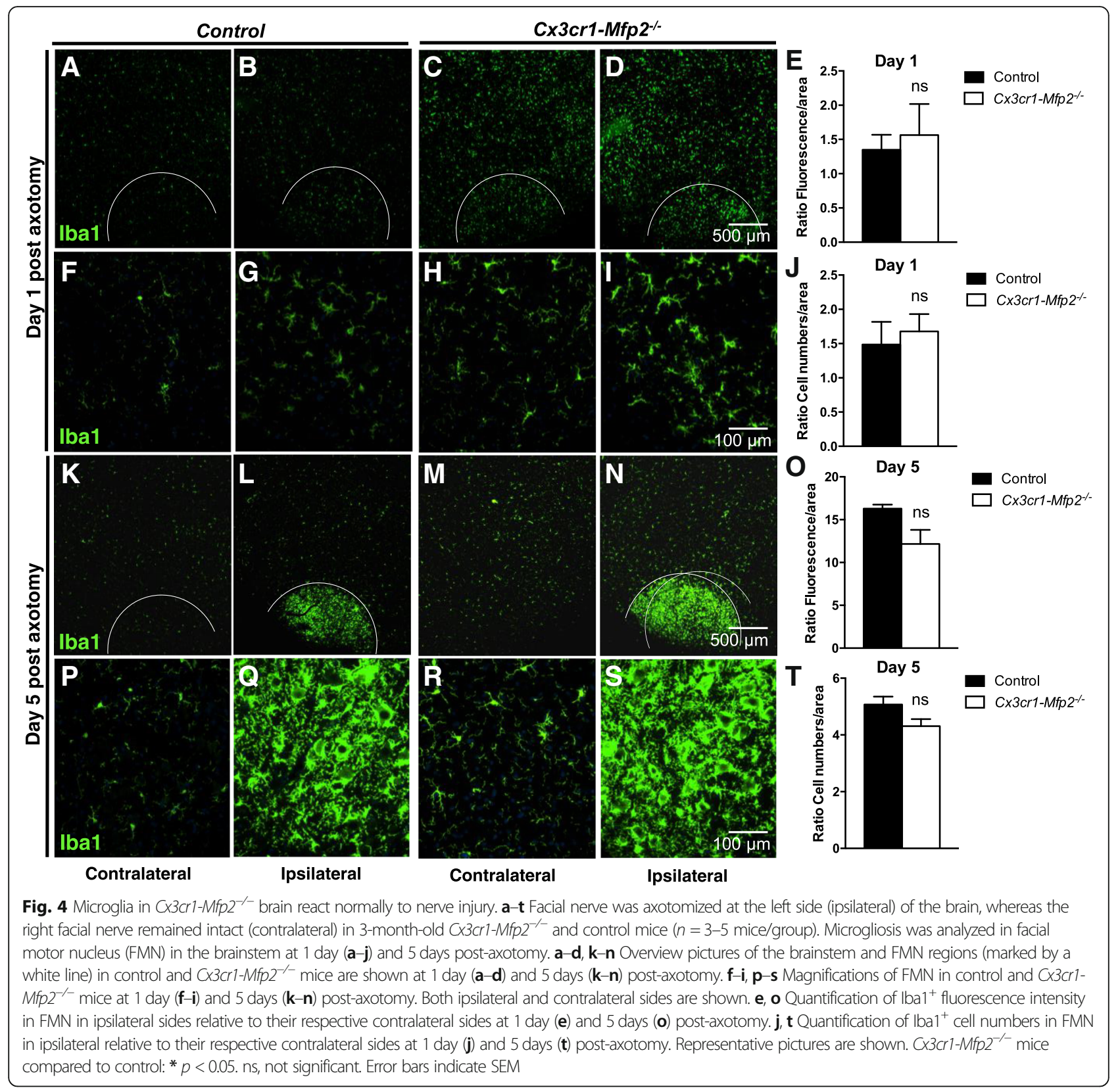

in $C \times 3 c r 1-M f p 2^{-/-}$versus control mice (Fig. 6c). These results demonstrate that neuronal signal transmission and amplitudes of brainstem responses are intact in C $x 3 \mathrm{cr} 1$ $M f p 2^{-/-}$mice.

Whereas constitutive $M f p 2^{-/-}$mice succumb before the age of 6 months and show severely impaired locomotor activity, exploration, and fear conditioning as an index of cognition at early age, the neural-specific Nestin-Mfp $2^{-/-}$mice survive up to 1 year and clinical impairments were delayed and less pronounced at the end stage of disease [11]. In order to investigate whether microglia-restricted loss of MFP2 affects grip strength, motor abilities, and behavior, the same tests were performed on Cx3cr1-Mfp $2^{-/-}$mice at the age of 8 months when inflammatory activation is manifested. We found that grip strength of front paws (Fig. 7a) and all paws together (Fig. 7b) remains intact in Cx3cr1-Mfp $2^{-/-}$ mice. Grip strength and coordination on an inverted grid did not differ in $C x 3 c r 1-M f p 2^{-/-}$mice and agematched control mice (Fig. 7c). Cx3cr1-Mfp $2^{-/-}$mice displayed normal locomotor activity in an open field environment as they display a similar amount of corner entries (Fig. 7d) and similar path length (Fig. 7e) as compared to control mice. $C \times 3 c r 1-M f p 2^{-/-}$mice exhibited a normal explorative behavior as the mean distance to the center (Fig. 7f), number of center entries (Fig. 7g), and 
lba1
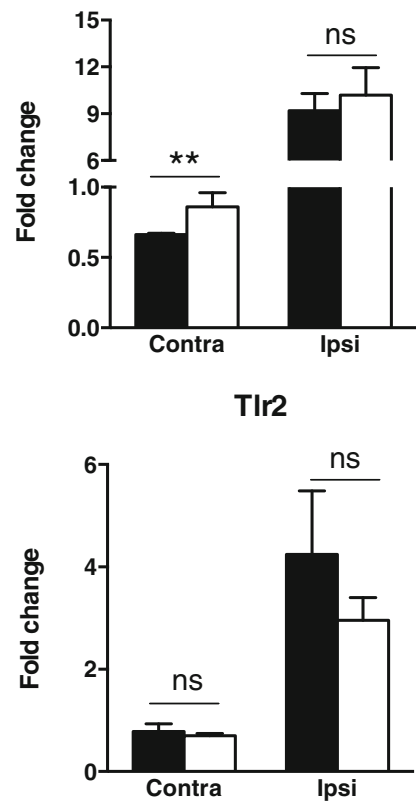

Csf1r

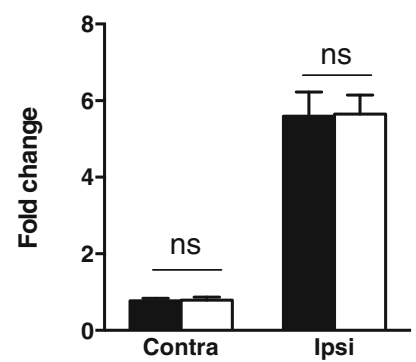

F4/80
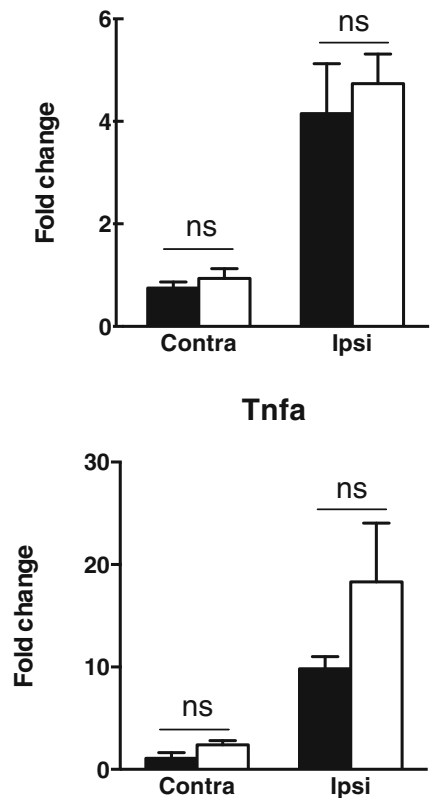

Tgfbr1

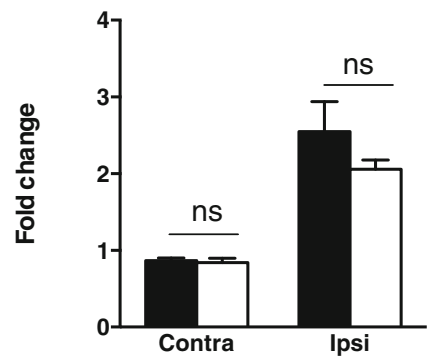

Tspo

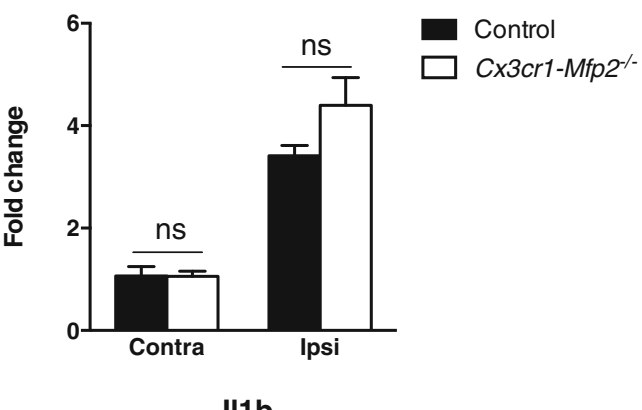

II1b

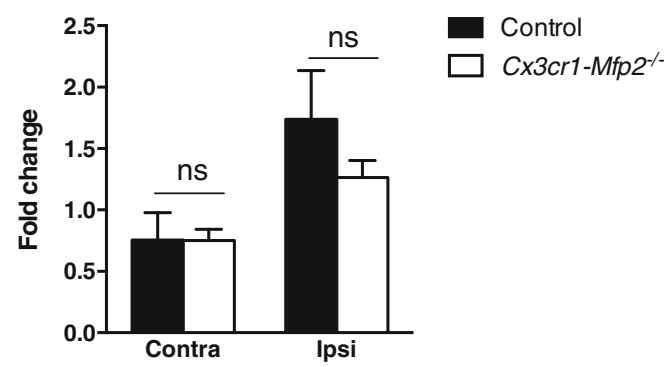

Fig. 5 Microglia in Cx3cr1-Mfp $2^{-/-}$brain adopt a normal inflammatory phenotype after nerve injury. Facial nerve was axotomized at the left side (ipsilateral) of the brain, whereas the right facial nerve remained intact in 3-month-old $\mathrm{C} \times 3 \mathrm{cr}$ - $-\mathrm{Mfp}^{-1-}$ and control. Transcript levels of inflammatory and microglial markers were analyzed in facial nuclei at 5 days post-axotomy. The expression of all markers significantly increased at the axotomized ipsilateral side (ipsi) of the brain versus intact contralateral side (contra) in both $\mathrm{C} \times 3 \mathrm{cr} 1-\mathrm{Mfp} 2^{-/-}$and control mice (significance levels not shown). There were no differences between genotypes at the affected ipsilateral side. CX3cr1-Mfp $2^{-1-}$ mice compared to control: ${ }^{*} p<0.05,{ }^{* *} p<0.01,{ }^{* * *} p<0.0001$. ns, not significant. $n=5$ mice/group. Error bars indicate SEM

time in the center (not shown) were similar to the control mice. Fear-conditioned memory was analyzed by passive avoidance test. There was no significant difference in time when $C x 3 c r 1-M f p 2^{-/-}$and control mice enter into the dark room during training (data not shown). During the test phase, $C \times 3 c r 1-M f p 2^{-/-}$and control mice showed a similar delay in time to enter the dark room (Fig. 7h), demonstrating that the 8-month-old Cx3cr1-Mfp $2^{-/-}$mice have a normal cognition. Taken together, we show that $M f p 2^{-/-}$microglia in a genetically intact brain environment do not induce abnormalities in motor function, explorative behavior, and cognition before 8 months of age.

\section{Discussion}

In this study, we investigated the impact of deletion of the pivotal peroxisomal $\beta$-oxidation enzyme MFP2 from microglia on immune response and neural functioning. Previous data demonstrated that global loss of MFP2 elicited an early-onset and extensive microgliosis in the CNS that was accompanied by quick deterioration of the mice $[2,10]$. Here, we established that $M f p 2^{-/-}$microglia in a genetically intact CNS environment adopt an inflammatory activated and proliferative state. Despite the pro-inflammatory microglial state, $C x 3 c r 1-M f p 2^{-/-}$ mice exhibited normal clinical performance and cognition. In addition, we found that acute inflammatory and 

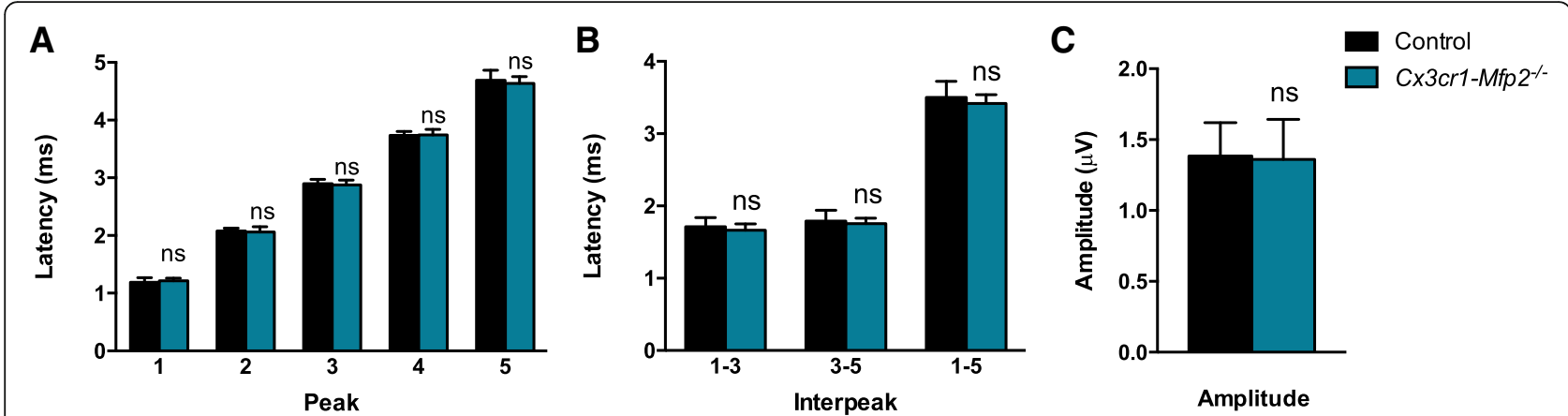

Fig. 6 Intact neuronal functioning in C $\times 3 \mathrm{cr} 1-\mathrm{Mfp}^{-/-}$brain. a-c BAEP test shows normal brainstem responses in C $\times 3 \mathrm{Cr} 1-\mathrm{Mfp} 2^{-/-}$versus control mice at 8 months of age. a Mean peak latencies show that all peaks assigned to specific brainstem regions (peak 2-4) and thalamus/cortex regions (peak 5) show normal latencies of the auditory signal in $\mathrm{C} \times 3 \mathrm{Cr} 1-\mathrm{Mfp} 2^{-/-}$mice. $\mathbf{b}$ Normal brain responses to the auditory stimulus were shown by similar interpeak latencies in CX3cr1-Mfp2 ${ }^{-/-}$and control mice. c Peak amplitudes are normal in CX3cr1-Mfp2 ${ }^{-/-}$versus control mice. $n=5-9$ mice/group. ns, not significant. Error bars indicate SEM

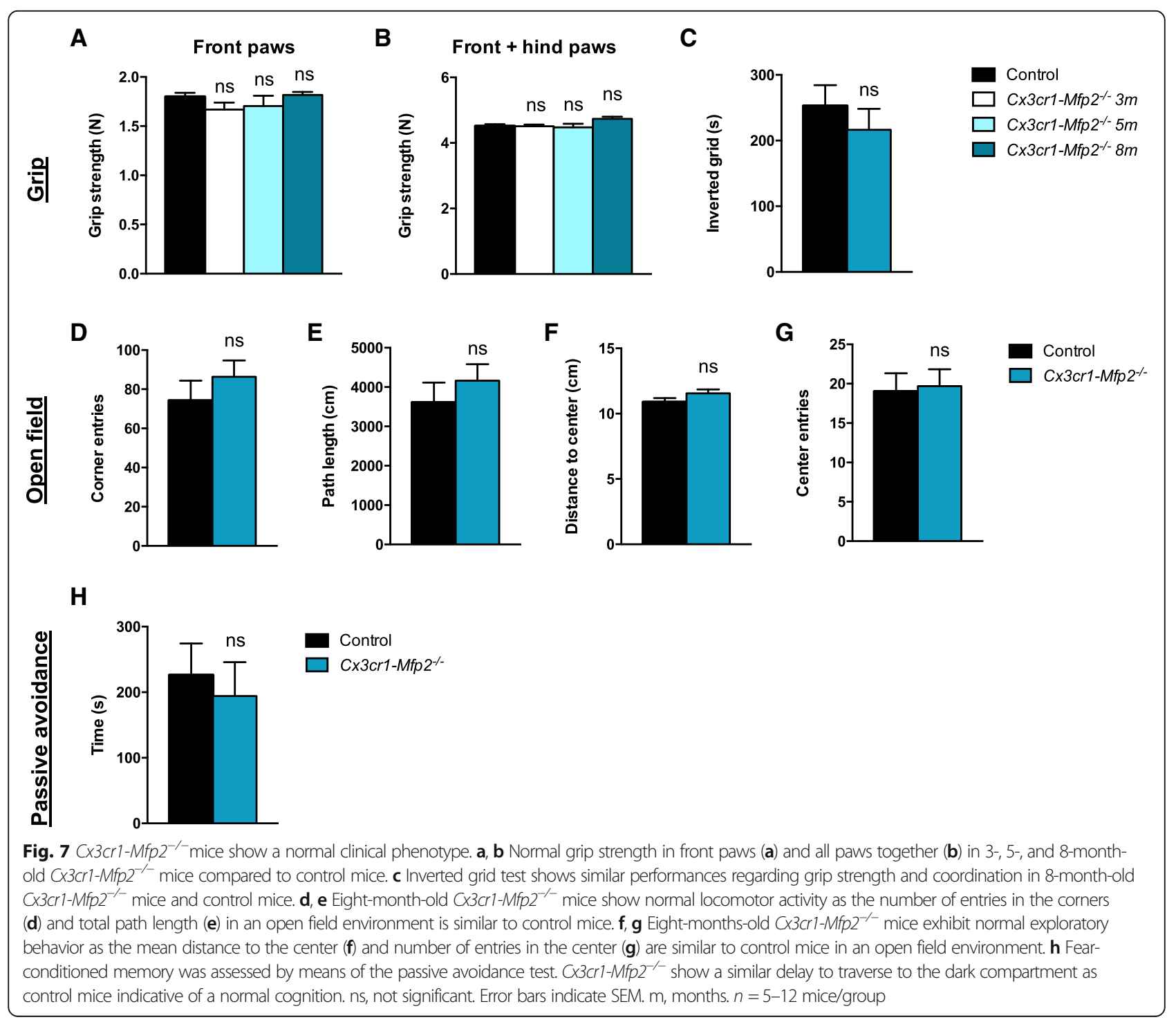


neuronal injury provoked normal responses of $M f p 2^{-/-}$ microglia in Cx3cr1-M $f p 2^{-/-}$mice during the post-injury period. Our data strongly suggest that MFP2 deficiency in microglia causes intrinsic pro-inflammatory deregulation, which is not harmful for neuronal function, motor function, and cognition in mice during their first year of life.

Histological examination of the Cx3cr1-M $f p 2^{-/-}$brain demonstrated progressive development of microgliosis in the absence of neuronal degeneration. Our results indicate that microglial proliferation is stimulated by IL-34 rather than by CSF1 signaling. The CSF1R is ligated by both CSF1 and IL-34 which play redundant roles in developing and adult brain [35]. It was demonstrated that IL-34 binding on CSF1R promotes maintenance and proliferation of microglia in the brain [36-39]. Although both IL-34 and CSF1 may induce microglial proliferation upon neurodegeneration, IL-34 has a stronger proliferationinducing capacity and higher expression in the brain compared to CSF1 $[35,40]$. Despite identical signaling pathways induced by CSF1 and IL-34 downstream CSF1R, a recent study found that IL-34-stimulated monocytes produce different cytokines/chemokines in an inflammatory context and exhibit a distinct polarization potential compared to CSF1-derived macrophages [41]. There is indeed increasing evidence that IL-34 expression is upregulated in pathological conditions and plays important roles in autoimmune disorders, infections, and inflammatory conditions [42]. Previous results showed that Il34 levels were also increased in constitutive $M f p 2^{-/-}$mice (eightfold) and neural-specific Nestin-Mfp $2^{-/-}$mice (fivefold) [11], whereas Csf1 levels were unchanged in both mouse models at the end stage of disease. Further research is however necessary to elucidate the distinct biological profile and effects of both cytokines.

The proliferation of microglia in Cx3cr1-Mfp $2^{-/-}$mice was accompanied with morphological transformation characterized by a swollen cell soma and thicker and shorter protrusions, both typical features of microglial activation $[15,43]$. A progressive expansion of $\mathrm{F} 4 / 80^{+}$cells in $\mathrm{Cx} 3 \mathrm{cr} 1$ $M f p 2^{-/-}$brains verified that increasing numbers of $M f p 2^{-/-}$ microglia are activated from at least 3 months of age. In contrast to $M f p 2^{-/-}$microglia in constitutive $M f p 2^{-/-}$mice, which adopt a mixed pro- and anti-inflammatory pheno-

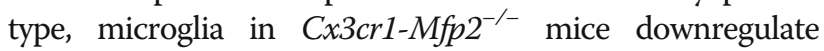
anti-inflammatory and induce pro-inflammatory cytokines from 8 months of age. This demonstrates that $M f p 2^{-/-}$ microglia in an intact CNS environment adopt a pro-inflammatory state several months after microglial proliferation and activation were initiated. These observations are in line with the pro-inflammatory profile of BV2 microglia in which another peroxisomal $\beta$-oxidation enzyme, ACOX1, was inactivated [44].

It should be noted that transient $C x 3 c r 1$ promoter activity has been detected in neurons during the development in some mouse models [45]. However, in $C \times 3 \operatorname{cr} 1-M f p 2^{-/-}$mice, we did not find evidence for the neuronal inactivation of MFP2 based on normal $M f p 2$ transcripts in non-microglial cells and the preservation of MFP2 activity in whole brain homogenates of C $x 3 \mathrm{cr} 1-\mathrm{M} f \mathrm{p} 2^{-/-}$mice. The latter is compatible with the fact that microglia only constitute approximately $10 \%$ of brain cells $[46,47]$ and that MFP2 expression in microglia is lower or similar to the more abundant cell types [48]. An additional argument supporting that the reactive microglial phenotype does not depend on the neuronal inactivation of MFP2 is the fact that neuronal deficits such as Purkinje cell degeneration were not observed in $C \times 3 c r 1-M f p 2^{-/-}$mice, in contrast to constitutive $M f p 2$ knockouts [2]. Finally, microglial proliferation and transformation were not only seen in gray but also in white matter regions.

We also examined how $M f p 2^{-/-}$microglia respond to acute inflammatory stimuli or to neuronal injury at an early disease stage, before inflammatory polarization was established. $M f p 2^{-/-}$microglia show normal responses to acute inflammatory stimuli in vitro and in vivo. $M f p 2^{-/-}$microglia acquired anti-inflammatory properties in response to an IL-4 stimulus which were indistinguishable from control microglia. An acute systemic pro-inflammatory stimulus elicited a similar response in microglia in Cx3cr1-Mfp $2^{-/-}$compared to control mice, proving that microglia in $C \times 3 c r 1-M f p 2^{-/-}$mice are not primed despite their inflammatory activated state in unstimulated conditions. In contrast, $M f p 2^{-/-}$microglia in $M f p 2^{-/-}$mice are primed in the absence of systemic inflammation, and neuronal transmission is severely disturbed, suggesting that dysfunctional microglia-neuron bidirectional communication might trigger microglial priming and rapid progression of disease in constitutive $M f p 2^{-\prime-}$ mice [11]. Neuronal injury was induced by unilateral axotomy of the facial nerve of $C x 3 c r 1-M f p 2^{-/-}$mice. We found that microglial responses in the axotomized relative to the intact FMN in $C \times 3 c r 1-M f p 2^{-/-}$and control brain were comparable. Similar expression levels of several proand anti-inflammatory molecules in the ipsilateral FMN of $C \times 3 c r 1-M f p 2^{-/-}$and control mice confirmed that $M f p 2^{-/-}$ microglia do not elicit an exaggerated response to nerve injury during the acute post-injury period. In conclusion, microglia in Cx3cr1-Mfp $2^{-/-}$mice respond properly to acute pro- and anti-inflammatory challenges and to neuronal injury.

Our previous study demonstrated clear differences in the neuropathology of constitutive $M f p 2^{-/-}$mice versus neural-specific Nestin-Mfp $2^{-/-}$mice that were paralleled by distinct microglial phenotypes, indicating that $M f p 2^{-/-}$ microglia play a role in the severe neuropathology of $M f p 2^{-/-}$mice [11]. However, we found that selective deletion of MFP2 from microglia in the Cx3cr1-Mfp $2^{-/-}$ 
brain did not affect grip strength, locomotor activity, explorative behavior, and fear conditioning as an index of cognitive function within the time frame wherein all $M f p 2^{-/-}$mice and Nestin-Mfp $2^{-/-}$mice have died from the disease [19]. Hence, the early-onset clinical and behavioral abnormalities in $M f p 2^{-/-}$mice can neither be assigned purely to neuronal deficits nor to intrinsic microglial pathology. Likewise, the BAEP test demonstrated that brainstem responses and peak amplitudes are normal in Cx3cr1$M f p 2^{-/-}$mice in contrast to affected responses and amplitudes in $M f p 2^{-/-}$mice and to a lesser extent in Nestin-Mfp $2^{-/-}$mice [11]. In the healthy brain, neurons persistently restrain microglia in order to maintain their surveilling state. Neurons in danger downregulate these restraint signals and send out "help me" signals that trigger microglial activation in pathological situations $[15,49,50]$. In $M f p 2^{-/-}$brain, the impaired neuronal signaling in the BAEP test was associated with lowered expression of the neuronal restraint signals $C x 3 c l$ and $C d 200$. In accordance to intact neuronal functioning in $C \times 3 c r 1-M f p 2^{-/-}$mice, the expression of these neuron-microglia signaling molecules was normal in Cx3cr1-Mfp $2^{-/-}$brain. This indicates that intrinsic microglial pathology by itself is not sufficient to cause early-onset dysfunctional neuronal transmission in $M f p 2^{-/-}$mice. Taking into account all data on microglial reactivity, neuronal functioning, and neuropathological features, we hypothesize that impaired functioning of the $\mathrm{CNS}$ and clinical deterioration in $M f p 2^{-/-}$deficiency occur through synergistic instability of distinct brain cell types in $M f p 2^{-/-}$mice.

\section{Conclusion}

We demonstrated in this study that $M f p 2^{-/-}$microglia in a genetically intact brain environment intrinsically adopt a proliferative and modified inflammatory state. The mild pro-inflammatory phenotype acquired by $M f p 2^{-/-}$microglia does not give rise to neuronal dysfunction nor to abnormal clinical behavior. Nevertheless, we cannot exclude that microglia become neurotoxic at a later stage. Our data indicate that microglia can develop a chronically proliferative and pro-inflammatory phenotype through cell-autonomous dysfunction without affecting the CNS environment and murine clinical behavior.

\section{Additional files}

Additional file 1: Figure S1. No microgliosis in both Cre-positive and Crenegative control mice. (A-D) No differences in microglia number and shape are observed between Cre-positive (Cre Mfp $\left.2^{\text {Wt LoxP }}\right)$ and Cre-negative (Mfp $2^{\text {Wt }}$ LoxP Control mice at 5 months of age (A-D) and 12 months of age (not shown). Representative pictures are shown. $n=3-5$ mice/group. (PPTX $988 \mathrm{~kb}$ )

Additional file 2: Figure S2. Efficient and selective inactivation of MFP2 in microglia in $\mathrm{C} \times 3 \mathrm{Cr} 1-\mathrm{Mfp}^{-/-}$mice. (A) Microglia were isolated from 11month-old control and $\mathrm{C} \times 3 \mathrm{Cr} 1-\mathrm{Mfp}^{-1-}$ mice, and microglia purity was confirmed by the high expression of microglial markers (Tmem119 and P2ry12) in the positive (microglia) versus the negative fraction (neurons, astrocytes, and oligodendrocytes). Transcript expression of Mfp2 was determined in control and $\mathrm{C} \times 3 \mathrm{Cr} 1-\mathrm{Mfp}^{-/-}$in both positive and negative fraction. Representative experiment out of two with similar results. (B) MFP2 activity in brain homogenates of control and $\mathrm{C} \times 3 \mathrm{Cr} 1-\mathrm{Mfp}^{-1-}$ mice. $n=2-3$ mice/group. Mean \pm SD is shown. (PPTX $46 \mathrm{~kb}$ )

Additional file 3: Figure S3. Inflammatory properties of cultured $\mathrm{Mfp}^{-/-}$ and control microglia. MACS-isolated microglia from P8 mice were kept either in basal conditions or polarized to a pro-inflammatory state (II1 $\beta /$ IFNY) or an anti-inflammatory state (IL4). Transcript expression of pro-inflammatory (Tnfa, iNOS, Cxcl1) and anti-inflammatory cytokines (Arg1, Fizz1, Ym1) were determined. Significance levels: $\Phi p<0.05, \Phi \Phi$ $p<0.01$, ФФФФ $p<0.0001$; ns, not significant. $n=8-11$ mice/group. (PPTX $59 \mathrm{~kb}$ )

\section{Abbreviations}

BAEP: Brainstem auditory evoked potentials; CNS: Central nervous system; CSF1R: Colony-stimulating factor 1 receptor; FMN: Facial motor nucleus; i.C.V.: Intracerebroventricular; IHC: Immunohistochemistry; II: Interleukin; LPS: Lipopolysaccharide; MFP2: Multifunctional protein 2; OF: Open field; PA: Passive avoidance

\section{Acknowledgements}

The authors are grateful to Steffen Jung (Weizmann Institute of Science, Rehovot, Israel) for generously providing (X3 cr1-Cre mice. They thank Yannick Das and Prof Paul Van Veldhoven for assistance with measuring MFP2 enzyme activity, and Benny Das and Ann Bouché for excellent technical assistance.

\section{Funding}

This work was funded by grants from Fonds Wetenschappelijk Onderzoek Vlaanderen (G.0675.12 and G.0A15.13), KU Leuven (OT12/78), and ERA-Net Neuron (MICRO-MET).

\section{Availability of data and materials}

All data generated or analysed during this study are included in this published article and its supplementary information files.

\section{Authors' contributions}

LB performed most of the experiments, analyzed and interpreted the data, and wrote the manuscript. SB assisted with the analysis of the facial nerve axotomy experiment. IG performed, analyzed, and reported the i.c.V. IL-4 experiment, microglia isolations, and culturing. SS and RH supervised the behavioral experiments. MB guided the project and was a major contributor in writing the manuscript. All authors read and approved the final manuscript.

\section{Ethics approval}

All experiments were conducted in accordance with "Guidelines for Care and Use of Experimental Animals" and fully approved by the Research Ethical committee of the KU Leuven (\#190/2012, \#181/2015).

\section{Consent for publication}

Not applicable

Competing interests

The authors declare that they have no competing interests.

\section{Publisher's Note}

Springer Nature remains neutral with regard to jurisdictional claims in published maps and institutional affiliations.

\section{Author details}

${ }^{1}$ Department of Pharmaceutical and Pharmacological Sciences, Laboratory for Cell Metabolism, KU Leuven - University of Leuven, Campus Gasthuisberg O/N2, Herestraat 49, B-3000 Leuven, Belgium. ²Faculty of Psychology and Educational Sciences, Biological Psychology Unit, KU Leuven - University of Leuven, B-3000 Leuven, Belgium. ${ }^{3}$ Department of Neurosciences, Laboratory for Neurobiology, KU Leuven - University of Leuven, Leuven, Belgium. ${ }^{4}$ Center for Brain and Disease Research, VIB, Leuven, Belgium. ${ }^{5}$ Neurology 
Department, University Hospitals Leuven, Leuven, Belgium. ${ }^{6}$ Present Address: Center for Translational and Computational Neuro-immunology, Department of Neurology, Columbia University Medical Center, New York City, NY, USA.

Received: 11 December 2018 Accepted: 24 February 2019

Published online: 13 March 2019

\section{References}

1. Huyghe $S$, Schmalbruch $H$, Hulshagen L, Veldhoven PV, Baes M, Hartmann D. Peroxisomal multifunctional protein-2 deficiency causes motor deficits and glial lesions in the adult central nervous system. Am J Pathol. 2006; 168(4):1321-34.

2. Verheijden S, Bottelbergs A, Krysko O, Krysko DV, Beckers L, De Munter S, Van Veldhoven PP, Wyns S, Kulik W, Nave KA, et al. Peroxisomal multifunctional protein-2 deficiency causes neuroinflammation and degeneration of Purkinje cells independent of very long chain fatty acid accumulation. Neurobiol Dis. 2013;58:258-69.

3. Ferdinandusse S, Denis S, Mooyer PA, Dekker C, Duran M, Soorani-Lunsing RJ, Boltshauser E, Macaya A, Gartner J, Majoie CB, et al. Clinical and biochemical spectrum of D-bifunctional protein deficiency. Ann Neurol. 2006:59(1):92-104.

4. Huyghe S, Mannaerts GP, Baes M, Van Veldhoven PP. Peroxisomal multifunctional protein-2: the enzyme, the patients and the knockout mouse model. Biochim Biophys Acta. 2006;1761(9):973-94.

5. Van Veldhoven PP. Biochemistry and genetics of inherited disorders of peroxisomal fatty acid metabolism. J Lipid Res. 2010;51(10):2863-95.

6. Lines MA, Jobling R, Brady L, Marshall CR, Scherer SW, Rodriguez AR, Lee L, Lang $A E$, Mestre TA, Wanders RJ, et al. Peroxisomal D-bifunctional protein deficiency: three adults diagnosed by whole-exome sequencing. Neurology. 2014:82(11):963-8.

7. Lieber DS, Hershman SG, Slate NG, Calvo SE, Sims KB, Schmahmann JD, Mootha VK. Next generation sequencing with copy number variant detection expands the phenotypic spectrum of HSD17B4-deficiency. BMC Med Genet. 2014:15:30

8. Khan A, Wei XC, Snyder FF, Mah JK, Waterham H, Wanders RJ. Neurodegeneration in D-bifunctional protein deficiency: diagnostic clues and natural history using serial magnetic resonance imaging. Neuroradiology. 2010; 52(12):1163-6.

9. Pierce SB, Walsh T, Chisholm KM, Lee MK, Thornton AM, Fiumara A, Opitz JM, Levy-Lahad E, Klevit RE, King MC. Mutations in the DBPdeficiency protein HSD17B4 cause ovarian dysgenesis, hearing loss, and ataxia of Perrault syndrome. Am J Hum Genet. 2010;87(2):282-8.

10. Verheijden S, Beckers L, Casazza A, Butovsky O, Mazzone M, Baes M. Identification of a chronic non-neurodegenerative microglia activation state in a mouse model of peroxisomal beta-oxidation deficiency. Glia. 2015;63(9):1606-20.

11. Beckers L, Stroobants S, D'Hooge R, Baes M. Neuronal dysfunction and behavioral abnormalities are evoked by neural cells and aggravated by inflammatory microglia in peroxisomal beta-oxidation deficiency. Front Cell Neurosci. 2018;12:136

12. Beckers L, Stroobants S, Verheijden S, West B, D'Hooge R, Baes M. Specific suppression of microgliosis cannot circumvent the severe neuropathology in peroxisomal beta-oxidation-deficient mice. Mol Cell Neurosci. 2017;80:123-33.

13. Davalos D, Grutzendler J, Yang G, Kim JV, Zuo Y, Jung S, Littman DR, Dustin ML, Gan WB. ATP mediates rapid microglial response to local brain injury in vivo. Nat Neurosci. 2005;8(6):752-8.

14. Wyss-Coray T, Mucke L. Inflammation in neurodegenerative disease--a double-edged sword. Neuron. 2002;35(3):419-32.

15. Saijo K, Glass CK. Microglial cell origin and phenotypes in health and disease. Nat Rev Immunol. 2011;11(11):775-87.

16. Crotti A, Ransohoff RM. Microglial physiology and pathophysiology: insights from genome-wide transcriptional profiling. Immunity. 2016;44(3):505-15.

17. Neumann $H$, Kotter MR, Franklin RJ. Debris clearance by microglia: an essential link between degeneration and regeneration. Brain. 2009; 132(Pt 2):288-95.

18. Michell-Robinson MA, Touil H, Healy LM, Owen DR, Durafourt BA, Bar-Or A, Antel JP, Moore CS. Roles of microglia in brain development, tissue maintenance and repair. Brain. 2015;138(Pt 5):1138-59.
19. Verheijden S, Beckers L, De Munter S, Van Veldhoven PP, Baes M. Centra nervous system pathology in MFP2 deficiency: insights from general and conditional knockout mouse models. Biochimie. 2014;98:119-26.

20. Yona S, Kim KW, Wolf Y, Mildner A, Varol D, Breker M, Strauss-Ayali D, Viukov S, Guilliams M, Misharin A, et al. Fate mapping reveals origins and dynamics of monocytes and tissue macrophages under homeostasis. Immunity. 2013; 38(1):79-91

21. Cardona AE, Pioro EP, Sasse ME, Kostenko V, Cardona SM, Dijkstra IM, Huang D, Kidd G, Dombrowski S, Dutta R, et al. Control of microglial neurotoxicity by the fractalkine receptor. Nat Neurosci. 2006;9(7):917-24.

22. Wolf $\mathrm{Y}$, Yona S, Kim KW, Jung S. Microglia, seen from the CX3CR1 angle. Front Cell Neurosci. 2013;7:26.

23. D'Hooge R, Lullmann-Rauch R, Beckers T, Balschun D, Schwake M, Reiss K, von Figura K, Saftig P. Neurocognitive and psychotiform behavioral alterations and enhanced hippocampal long-term potentiation in transgenic mice displaying neuropathological features of human alphamannosidosis. J Neurosci. 2005;25(28):6539-49.

24. Pepe G, Calderazzi G, De Maglie M, Villa AM, Vegeto E. Heterogeneous induction of microglia M2a phenotype by central administration of interleukin-4. J Neuroinflammation. 2014;11:211.

25. Beel S, Moisse M, Damme M, De Muynck L, Robberecht W, Van Den Bosch $L$, Saftig P, Van Damme P. Progranulin functions as a cathepsin D chaperone to stimulate axonal outgrowth in vivo. Hum Mol Genet. 2017; 26(15):2850-63.

26. Hulshagen L, Krysko O, Bottelbergs A, Huyghe S, Klein R, Van Veldhoven PP, De Deyn PP, D'Hooge R, Hartmann D, Baes M. Absence of functional peroxisomes from mouse CNS causes dysmyelination and axon degeneration. J Neurosci. 2008;28(15):4015-27.

27. Bottelbergs A, Verheijden S, Van Veldhoven PP, Just W, Devos R, Baes M. Peroxisome deficiency but not the defect in ether lipid synthesis causes activation of the innate immune system and axonal loss in the central nervous system. J Neuroinflammation. 2012;9:61.

28. Livak KJ, Schmittgen TD. Analysis of relative gene expression data using real-time quantitative PCR and the 2(-Delta Delta C(T)) method. Methods. 2001;25(4):402-8.

29. Baes M, Huyghe S, Carmeliet P, Declercq PE, Collen D, Mannaerts GP, Van Veldhoven PP. Inactivation of the peroxisomal multifunctional protein-2 in mice impedes the degradation of not only 2-methyl-branched fatty acids and bile acid intermediates but also of very long chain fatty acids. J Biol Chem. 2000;275(21):16329-36.

30. De Munter S, Bamps D, Malheiro AR, Kumar Baboota R, Brites P, Baes M. Autonomous Purkinje cell axonal dystrophy causes ataxia in peroxisomal multifunctional protein-2 deficiency. Brain Pathol. 2018;28(5):631-43.

31. Moran LB, Graeber MB. The facial nerve axotomy model. Brain Res Brain Res Rev. 2004:44(2-3):154-78.

32. Tay TL, Mai D, Dautzenberg J, Fernandez-Klett F, Lin G, Sagar DM, Drougard A, Stempfl T, Ardura-Fabregat A, et al. A new fate mapping system reveals context-dependent random or clonal expansion of microglia. Nat Neurosci. 2017;20(6):793-803.

33. Krasemann S, Madore C, Cialic R, Baufeld C, Calcagno N, El Fatimy R, Beckers L, O'Loughlin E, Xu Y, Fanek Z, et al. The TREM2-APOE pathway drives the transcriptional phenotype of dysfunctional microglia in neurodegenerative diseases. Immunity. 2017:47(3):566-81 e569.

34. Singer $W$, Panford-Walsh $\mathrm{R}$, Knipper $M$. The function of BDNF in the adult auditory system. Neuropharmacology. 2014;76(Pt C):719-28.

35. Wei S, Nandi S, Chitu V, Yeung YG, Yu W, Huang M, Williams LT, Lin H, Stanley ER. Functional overlap but differential expression of CSF-1 and IL-34 in their CSF-1 receptor-mediated regulation of myeloid cells. J Leukoc Biol. 2010;88(3):495-505.

36. Zelante T, Ricciardi-Castagnoli P. The yin-yang nature of CSF1R-binding cytokines. Nat Immunol. 2012;13(8):717-9.

37. Greter M, Lelios I, Pelczar P, Hoeffel G, Price J, Leboeuf M, Kundig TM, Frei K, Ginhoux F, Merad M, et al. Stroma-derived interleukin-34 controls the development and maintenance of langerhans cells and the maintenance of microglia. Immunity. 2012;37(6):1050-60.

38. Mizuno T, Doi $Y$, Mizoguchi $H$, Jin $S$, Noda M, Sonobe $Y$, Takeuchi $H$, Suzumura A. Interleukin-34 selectively enhances the neuroprotective effects of microglia to attenuate oligomeric amyloid-beta neurotoxicity. Am J Pathol. 2011;179(4):2016-27.

39. Wang Y, Szretter KJ, Vermi W, Gilfillan S, Rossini C, Cella M, Barrow AD, Diamond MS, Colonna M. IL-34 is a tissue-restricted ligand of CSF1R 
required for the development of Langerhans cells and microglia. Nat Immunol. 2012;13(8):753-60.

40. Gomez-Nicola D, Fransen NL, Suzzi S, Perry VH. Regulation of microglial proliferation during chronic neurodegeneration. J Neurosci. 2013;33(6): 2481-93.

41. Boulakirba S, Pfeifer A, Mhaidly R, Obba S, Goulard M, Schmitt T, Chaintreuil P, Calleja A, Furstoss N, Orange F, et al. LL-34 and CSF-1 display an equivalent macrophage differentiation ability but a different polarization potential. Sci Rep. 2018;8(1):256

42. Baghdadi M, Umeyama Y, Hama N, Kobayashi T, Han N, Wada H, Seino KI. Interleukin-34, a comprehensive review. J Leukoc Biol. 2018;104(5):931-51.

43. Salter MW, Beggs S. Sublime microglia: expanding roles for the guardians of the CNS. Cell. 2014;158(1):15-24.

44. Raas Q, Saih FE, Gondcaille C, Trompier D, Hamon Y, Leoni V, Caccia C, Nasser B, Jadot M, Menetrier F, et al. A microglial cell model for acyl-CoA oxidase 1 deficiency. Biochim Biophys Acta Mol Cell Biol Lipids. 2018;1864(4):567-76.

45. Haimon Z, Volaski A, Orthgiess J, Boura-Halfon S, Varol D, Shemer A, Yona S, Zuckerman B, David E, Chappell-Maor L, et al. Re-evaluating microglia expression profiles using RiboTag and cell isolation strategies. Nat Immunol. 2018;19(6):636-44.

46. Lawson LJ, Perry VH, Dri P, Gordon S. Heterogeneity in the distribution and morphology of microglia in the normal adult mouse brain. Neuroscience. 1990;39(1):151-70.

47. Pelvig DP, Pakkenberg H, Stark AK, Pakkenberg B. Neocortical glial cell numbers in human brains. Neurobiol Aging. 2008;29(11):1754-62.

48. Zhang Y, Chen K, Sloan SA, Bennett ML, Scholze AR, O'Keeffe S, Phatnani HP, Guarnieri P, Caneda C, Ruderisch N, et al. An RNA-sequencing transcriptome and splicing database of glia, neurons, and vascular cells of the cerebral cortex. J Neurosci. 2014;34(36):11929-47.

49. Polazzi E, Monti B. Microglia and neuroprotection: from in vitro studies to therapeutic applications. Prog Neurobiol. 2010;92(3):293-315.

50. Li Y, Du XF, Liu CS, Wen ZL, Du JL. Reciprocal regulation between resting microglial dynamics and neuronal activity in vivo. Dev Cell. 2012;23(6):1189-202.

Ready to submit your research? Choose BMC and benefit from:

- fast, convenient online submission

- thorough peer review by experienced researchers in your field

- rapid publication on acceptance

- support for research data, including large and complex data types

- gold Open Access which fosters wider collaboration and increased citations

- maximum visibility for your research: over $100 \mathrm{M}$ website views per year

At $\mathrm{BMC}$, research is always in progress.

Learn more biomedcentral.com/submissions 